\title{
Dynamic Behavior Analysis of Tethered Satellite System Based on Floquet Theory
}

\author{
Guangnan Zhu ${ }^{\mathrm{a}}$, Kuan Lu ${ }^{\mathrm{b}, *}$, Qingjie $\mathrm{Cao}^{\mathrm{a}}$, Panfeng Huang ${ }^{\mathrm{b}}$, Kangyu Zhang ${ }^{\mathrm{b}}$ \\ ${ }^{a}$ School of Astronautics, Harbin Institute of Technology, Harbin 150001, China \\ ${ }^{b}$ Northwestern Polytechnical University, Xi'an, 710072, PR China
}

\begin{abstract}
In this paper, an $n$-star general dynamic model of tethered satellite system with closed-loop configuration is provided. An analytical method for periodic solution stability of the general dynamic model is proposed based on Floquet theory, which proved that the periodic solution stability of the system depends on the maximum modulus for the eigenvalue of a matrix related to the Jacobian matrix. The periodic solution stability of a 3-star system with equilateral triangle as the initial configuration is analyzed as an example based upon the analytical method. The critical stable spin angular velocity of the 3-star system is analyzed when the system spins clockwise, and its numerical simulation is carried out to verify the results. The results show that the analytical method of periodic solution stability can solve the critical stable spin angular velocity accurately of the tethered satellite system, and the 3-star system can guarantee stable spin when the spin angular velocity is about 2.1 times of its revolution angular velocity, otherwise the disturbed system will not be able to re-converge to the initial configuration in finite time.
\end{abstract}

Keywords: Tethered satellite system, Floquet theory, Nonlinear dynamics

\footnotetext{
* Corresponding author

Email address: lukuan@nwpu.edu.cn (Kuan Lu)
} 


\section{Introduction}

Study on tethered satellite system (TSS) has become one central issue of concerns in spacecraft engineering, attracting the attention of researchers in a series of areas 1, 2. Since the first proposal of TSS, it has been extensively

5 applied in actual engineering, such as tethered space robot [3], tethered space harpoon [4], and tethered space net [5], etc. The first tethered satellite system mission TSS-1 was jointly proposed by the United States and Italy in 1992 [ [ Since then, many research institutions have carried out a large number of space experiments on tethered satellite systems [7, and made abundant achievements 8, 9, 10, 11, but also ushered in several mission failures [6, 12, 13].

Based upon this, most researchers concentrated on mechanism design and accordingly the dynamics and control of space capture [14. An analytical tether length rate control law for the deployment of a flexible TSS under the space perturbations was proposed [15]. Ref. [16] proposed a novel adaptive dynamic sliding mode scheme for the deployment of the tethered satellite system. Although the input and command signals are limited based on the strictly bounded terms, the control tension force is discontinuous. Another controller is designed by utilizing small-gain theorem in Ref. [17, while the transversal thrust is considered to suppress the tethers oscillation. A feedback tension control law for the deployment of space tether with tension constraint and saturation function is proposed by in Ref. [18. Sakawa [19] used the optimal control to solve the linearized nonlinear system. An input shaping scheme was proposed to generate reasonable planning of the control signal [20], and it is widely used to solve underactuated problem. An earth-oriented double-pyramid-formation was investigated in Ref. 21] and discovered that the stable configuration was along the nadir direction, and the optimal control of a double-pyramid Earth-facing formation was discussed. A novel adaptive dynamic sliding mode scheme for the deployment of the TSS is proposed in Ref. [15. Because of the Coriolis force and orbital motion, a fast and stable deployment/retrieval of the TSS is difficult to achieve 22 . 
Stability analysis is a core research content in mechanism study of vibration behaviors of dynamic systems. Many stability analysis methods have been used to study nonlinear vibration behaviors of tethered satellite system. The Floquet theory [23, 24] was used to solve a series of dynamic problems [25, 26], such as rotor dynamics [27, 28, few researchers used the Floquet theory to study the stability and dynamic behaviors of TSS.

The motivation of this paper is to analyze the dynamic behavior of the tethered satellite system based on Floquet theory. In section 2, a general model of tethered satellite system with closed loop configuration is established and a brief introduction to Floquet theory is also discussed. Section 3 proposes general method for stability analysis based upon the Floquet theory of the tethered satellite system and discusses the numerical results of an example based on ternary system. Finally, conclusions and outlooks are drawn in section 4.

\section{Dynamic Modeling of Tethered Satellite System}

\subsection{General Tethered Satellite System}

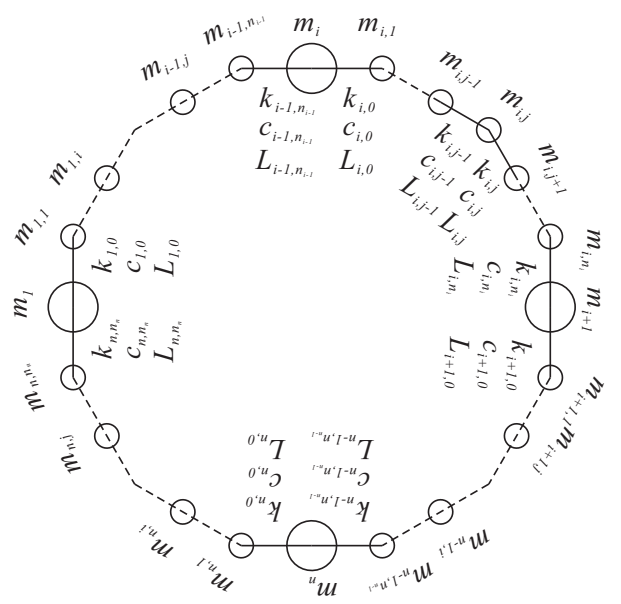

Figure 1: General tethered satellite system model.

A general model of a tethered satellite system with $n$ satellites $S_{1}, S_{2}, \cdots$, $S_{n}$ connected in a closed loop configuration is considered. The mathematical 
model of the tethered satellite system can be approximated to the bead-springdamping ring form shown in Fig. 1, no matter the mass of ropes are considered or not, by the following fixed steps.

1. Define $m_{i}$ as the mass of the $i$ th satellite $S_{i}$;

2. The $i$ th mass cable connecting satellite $S_{i}$ and $S_{i+1}$ is divided into a finite segment $\left(n_{i}\right)$ units consisting of concentrated mass, massless spring and damping, in which $1 \leq i<n$;

55 3. The mass $m_{i, j}$ of each unit at the $i$ th segment is defined in which $1 \leq j \leq n_{i}$; and spring stiffness $k_{i, j}$, damping coefficient $C_{i, j}$, and equilibrium length of each spring $L_{i, j}$, is defined in which $0 \leq j \leq n_{i}$, based upon the actual rope design parameters.

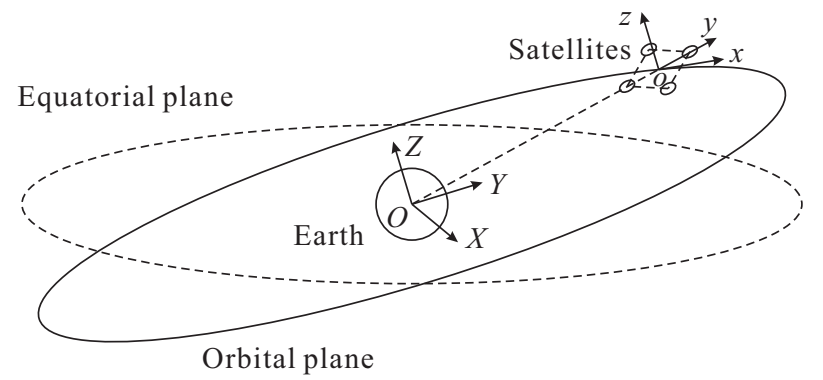

Figure 2: Schematic diagram of reference frame.

60 coordinate system $O-X Y Z$ is established by connected to the centroid of the earth fixedly, and the rotating coordinate system $o-x y z$ is established by connected to the centroid of the tethered satellite system fixedly, respectively, shown in Fig. 2 The positive direction of $X$-axis and $Z$-axis is defined as the direction along the centroid of the earth to the ascending node and the centroid of the earth to the north pole, respectively, and the positive direction of $Y$-axis is determined by the right hand rule, in the inertial coordinate system $O-X Y Z$. The positive direction of $y$-axis and $z$-axis is defined as the direction along the centroid of the earth to the centroid of the tethered satellite system and the 
same direction of the $Z$-axis, respectively, and the positive direction of $x$-axis is determined by the right hand rule, in the inertial coordinate system $o-x y z$. Assuming the revolution angular velocity of the $o-x y z$ around the $O-X Y Z$ is $\Omega$, and the positive direction of rotation is counterclockwise.

The motion equation of the $i$ th bead of the bead-spring-damping ring system in the coordinate $o-x y z$ can be expressed as follows

$$
m_{i} \ddot{\boldsymbol{r}}_{\boldsymbol{i}}=\boldsymbol{G}_{\boldsymbol{i}}+\boldsymbol{T}_{\boldsymbol{i}}+\boldsymbol{D}_{\boldsymbol{i}}+\boldsymbol{F}_{\boldsymbol{i}}^{\boldsymbol{e}}+\boldsymbol{F}_{\boldsymbol{i}}^{\boldsymbol{k}}
$$

in which $1 \leq i \leq n+m$ (including all the lumped mass of $n$ satellites and $m=$ ${ }_{75} \sum_{i=1}^{n-1} n_{i}$ tether units), and for the $i$ th bread, $m_{i}$ : the mass, $\boldsymbol{r}_{\boldsymbol{i}}=\left(x_{i}, y_{i}, j_{i}\right)^{\mathrm{T}}$ : the position vector in the $o-x y z, \boldsymbol{G}_{\boldsymbol{i}}$ : the gravity, $\boldsymbol{T}_{\boldsymbol{i}}$ : the rope tension, $\boldsymbol{D}_{\boldsymbol{i}}$ : the damping force, $\boldsymbol{F}_{\boldsymbol{i}} \boldsymbol{e}$ : the convected inertial force, $\boldsymbol{F}_{\boldsymbol{i}}^{\boldsymbol{k}}$ : the Coriolis inertial force, $\dot{\boldsymbol{r}}_{\boldsymbol{i}}$ and $\ddot{\boldsymbol{r}}_{\boldsymbol{i}}$ : the first and second order derivative of the position vector $\boldsymbol{r}_{\boldsymbol{i}}$ to the time $t$, respectively, without considering the perturbation factors such as $J_{2}$ perturbation, thermal effect, and atmospheric resistance.

Firstly, the gravitational term in Eq. (1) can be obtained as follows

$$
\boldsymbol{G}_{\boldsymbol{i}}=m_{i} \boldsymbol{g}_{\boldsymbol{i}}
$$

in which $\boldsymbol{g}_{\boldsymbol{i}}$ is the gravitational acceleration at the $i$ th bead provided by the earth, which can be expressed as follows

$$
\boldsymbol{g}_{\boldsymbol{i}}=-\frac{\mu_{E}\left(\boldsymbol{r}_{\boldsymbol{i}}-\boldsymbol{r}_{\boldsymbol{E}}\right)}{\left|\boldsymbol{r}_{\boldsymbol{i}}-\boldsymbol{r}_{\boldsymbol{E}}\right|^{3}}
$$

where $\mu_{E} \approx 3.986 \times 10^{5} \mathrm{~km}^{3} / \mathrm{s}^{2}$ is the gravity parameter of the earth, $\boldsymbol{r}_{\boldsymbol{E}}=$ $(0,-R, 0)^{\mathrm{T}}$ is the centroid position vector of the earth in $o-x y z, R=H+R_{E}$, in which $H$ is the orbital height of the BSDR system, and $R_{E}=6378 \mathrm{~km}$ is the average radius of the earth. Substituting Eq. (3) into Eq. (2), and the gravitational term of the $i$ th bead can be rewritten as follows

$$
\boldsymbol{G}_{\boldsymbol{i}}=-\frac{m_{i} \mu_{E}\left(\boldsymbol{r}_{\boldsymbol{i}}-\boldsymbol{r}_{\boldsymbol{E}}\right)}{\left|\boldsymbol{r}_{\boldsymbol{i}}-\boldsymbol{r}_{\boldsymbol{E}}\right|^{3}}
$$

Next, the tension term of tether in Eq. (1) can be obtained as follows $\boldsymbol{T}_{\boldsymbol{i}}=-s_{i, j} E_{i, j} A_{i, j}\left(\frac{1}{L_{i, j}}-\frac{1}{r_{i, j}}\right)\left(\boldsymbol{r}_{\boldsymbol{i}}-\boldsymbol{r}_{\boldsymbol{j}}\right)-s_{i, k} E_{i, k} A_{i, k}\left(\frac{1}{L_{i, k}}-\frac{1}{r_{i, k}}\right)\left(\boldsymbol{r}_{\boldsymbol{i}}-\boldsymbol{r}_{\boldsymbol{k}}\right)$, 
where

$$
\left\{\begin{array}{l}
j=2, k=n+m, \quad i=1, \\
j=1, k=n+m-1, \quad i=n+m \\
j=i+1, k=i-1, \quad \text { else. }
\end{array}\right.
$$

where $r_{i, j}=\left|\boldsymbol{r}_{\boldsymbol{i}}-\boldsymbol{r}_{\boldsymbol{j}}\right|$ is the instantaneous distance between the $i$ th and $j$ th bead and $s_{i, j}$ satisfies the following relation

$$
s_{i, j}= \begin{cases}1, & r_{i, j}>L_{i, j}, \\ 0, & \text { else },\end{cases}
$$

which control the effective of the tether tension.

Considering the damping term in Eq. (1) obtained as the following expression

$$
\boldsymbol{D}_{\boldsymbol{i}}=-s_{i, j} C_{i, j} \frac{\dot{r}_{i, j}}{r_{i, j}}\left(\boldsymbol{r}_{\boldsymbol{i}}-\boldsymbol{r}_{\boldsymbol{j}}\right)-s_{i, k} C_{i, k} \frac{\dot{r}_{i, k}}{r_{i, k}}\left(\boldsymbol{r}_{\boldsymbol{i}}-\boldsymbol{r}_{\boldsymbol{k}}\right),
$$

where $\dot{r}_{i, j}$ is the instantaneous relative speed between the $i$ th and $j$ th bead.

The convected inertial force term in Eq. (1) can be considered to be consisted with the translation convected inertial force and the centrifugal convected inertial force, which can be expressed as follows

$$
\boldsymbol{F}_{\boldsymbol{i}}^{\boldsymbol{e}}=-m_{i} \dot{\omega} \times\left(\boldsymbol{r}_{\boldsymbol{i}}-\boldsymbol{r}_{\boldsymbol{E}}\right)-m_{i} \omega \times\left(\omega \times\left(\boldsymbol{r}_{\boldsymbol{i}}-\boldsymbol{r}_{\boldsymbol{E}}\right)\right),
$$

where $\Omega$ is the value of spin angular velocity of the coordinate $o-x y z$ which equals to its revolution angular velocity numerically, and $\omega=(0,0, \Omega)^{\mathrm{T}}$ and $\dot{\omega}$ represent the rotational angular velocity and angular acceleration of the $o-x y z$. $\dot{\omega}=0$ must be hold by supposing the orbit of revolution is circular, then the relation can be obtained as follows

$$
-m_{i} \omega \times\left(\omega \times\left(\boldsymbol{r}_{\boldsymbol{i}}-\boldsymbol{r}_{\boldsymbol{E}}\right)\right)=\left[\begin{array}{ccc}
\boldsymbol{i} & \boldsymbol{j} & \boldsymbol{k} \\
0 & 0 & \Omega \\
-\left(y_{i}+R\right) \Omega & x_{i} \Omega & 0
\end{array}\right]=\left[\begin{array}{c}
-x_{i} \Omega^{2} \\
-\left(y_{i}+R\right) \Omega^{2} \\
0
\end{array}\right],
$$

where $\boldsymbol{i}, \boldsymbol{j}$, and $\boldsymbol{k}$ represent the $x, y$, and $z$ axis unit vectors in the $o-x y z$. Furthermore, the convected inertial force can be repressed as

$$
\boldsymbol{F}_{\boldsymbol{i}}^{\boldsymbol{e}}=\left(m_{i} x_{i} \Omega^{2}, m_{i}\left(y_{i}+R\right) \Omega^{2}, 0\right)^{\mathrm{T}} .
$$


Finally, consider the Coriolis inertia force term in Eq. (1) can be obtained as

$$
\boldsymbol{F}_{\boldsymbol{i}}^{\boldsymbol{k}}=-2 m_{i} \bar{\omega} \times \dot{\boldsymbol{r}}_{\boldsymbol{i}}
$$

where $\bar{\omega}=\omega$ is the spin angular velocity of the $o-x y z$, Eq. 12 can be rewritten as

$$
\boldsymbol{F}_{\boldsymbol{i}}^{\boldsymbol{k}}=\left(2 m_{i} \Omega \dot{y}_{i},-2 m_{i} \Omega \dot{x}_{i}, 0\right)^{\mathrm{T}}
$$

\subsection{Brief Introduction to Floquet Theory}

Floquet Theory is a stability theory for a periodic variable coefficient linear ordinary differential equation, which was put forward by G. Floquet in 1868 . This section will discuss the general method of $N$ dimensions equation. Consider a system of first order differential equations

$$
\dot{\boldsymbol{p}}(t)=\boldsymbol{A}(t) \boldsymbol{p}(t),
$$

where $\boldsymbol{A}$ is matrix with period $T$. Suppose the system (14) has $N$ of linearly independent particular solutions

$$
\left\{\begin{array}{l}
\boldsymbol{p}_{\mathbf{1}}(t)=\left(p_{11}(t), p_{12}(t), \cdots, p_{1 N}(t)\right)^{\mathrm{T}} \\
\boldsymbol{p}_{\mathbf{2}}(t)=\left(p_{21}(t), p_{22}(t), \cdots, p_{2 N}(t)\right)^{\mathrm{T}} \\
\vdots \\
\boldsymbol{p}_{\boldsymbol{N}}(t)=\left(p_{N 1}(t), p_{N 2}(t), \cdots, p_{N N}(t)\right)^{\mathrm{T}}
\end{array}\right.
$$

and the initial conditions are satisfied

$$
\left(\boldsymbol{p}_{1}(0), \boldsymbol{p}_{\mathbf{2}}(0), \cdots, \boldsymbol{p}_{\boldsymbol{N}}(0)\right)=\boldsymbol{I} .
$$

It is easy to prove that this set of particular solution is the fundamental solution system of system (14). Based on periodicity, if $\boldsymbol{P}_{\boldsymbol{i}}(t)(1 \leq i \leq N)$ is one of the particular solution, then $\boldsymbol{P}_{\boldsymbol{i}}(t+T)(1 \leq i \leq N)$ must be another one of the particular solution either, which can be expressed as

$$
p_{i j}(t+T)=\sum_{k=1}^{N} a_{i k} p_{k j}(t),
$$


in which $(1 \leq i, j \leq N)$. Substituting $t=0$ and Eq. (16) into Eq. (17), the equation can be solved as follows

$$
a_{i j}=p_{i j}(T) .
$$

In order to determine the stability of the periodic solution of the system (14), we hope to find a particular solution which satisfies the following conditions

$$
\boldsymbol{p}^{*}(t+T)=\rho \boldsymbol{p}^{*}(t)
$$

in which $\boldsymbol{p}^{*}(t)$ and $\boldsymbol{p}^{*}(t+T)$ can be expressed as the fundamental solution system (14)

$$
\boldsymbol{p}^{*}(t)=\left(\sum_{i=1}^{N} b_{i} p_{i 1}(t), \sum_{i=1}^{N} b_{i} p_{i 2}(t), \cdots, \sum_{i=1}^{N} b_{i} p_{i N}(t)\right)^{\mathrm{T}},
$$

and

$$
\boldsymbol{p}^{*}(t+T)=\left(\sum_{i=1}^{N} b_{i} p_{i 1}(t+T), \sum_{i=1}^{N} b_{i} p_{i 2}(t+T), \cdots, \sum_{i=1}^{N} b_{i} p_{i N}(t+T)\right)^{\mathrm{T}},
$$

respectively. It can be obtained by utilizing Eq. (17), Eq. (20), and Eq. 21) into Eq. $[19]$ as follows

$$
\rho\left[\begin{array}{c}
\sum_{i=1}^{N} b_{i} p_{i 1}(t) \\
\sum_{i=1}^{N} b_{i} p_{i 2}(t) \\
\vdots \\
\sum_{i=1}^{N} b_{i} p_{i N}(t)
\end{array}\right]=\left[\begin{array}{c}
\sum_{i=1}^{N} b_{i} p_{i 1}(t+T) \\
\sum_{i=1}^{N} b_{i} p_{i 2}(t+T) \\
\vdots \\
\sum_{i=1}^{N} b_{i} p_{i N}(t+T)
\end{array}\right]=\left[\begin{array}{c}
\sum_{i=1}^{N} b_{i} \sum_{j=1}^{N} a_{i j} p_{j 1}(t) \\
\sum_{i=1}^{N} b_{i} \sum_{j=1}^{N} a_{i j} p_{j 2}(t) \\
\vdots \\
\sum_{i=1}^{N} b_{i} \sum_{j=1}^{N} a_{i j} p_{j N}(t)
\end{array}\right] .
$$

The Eq. 22 can be rewritten as follows

$$
\boldsymbol{P}(t) \cdot \boldsymbol{M} \equiv \mathbf{0},
$$

assume that

$$
\boldsymbol{P}(t)=\left(\boldsymbol{p}_{\mathbf{1}}(t), \boldsymbol{p}_{\mathbf{2}}(t), \cdots, \boldsymbol{p}_{\boldsymbol{N}}(t)\right),
$$


and

$$
\boldsymbol{M}=\left[\begin{array}{c}
\left(a_{11}-\rho\right) b_{1}+a_{21} b_{2}+\cdots+a_{N 1} b_{N} \\
a_{12} b_{1}+\left(a_{22}-\rho\right) b_{2}+\cdots+a_{N 2} b_{N} \\
\vdots \\
a_{1 N} b_{1}+a_{2 N} b_{2}+\cdots+\left(a_{N N}-\rho\right) b_{N}
\end{array}\right],
$$

which means that the following relation must be held

$$
\left\{\begin{array}{l}
\left(a_{11}-\rho\right) b_{1}+a_{21} b_{2}+\cdots+a_{N 1} b_{N}=0 \\
a_{12} b_{1}+\left(a_{22}-\rho\right) b_{2}+\cdots+a_{N 2} b_{N}=0 \\
\vdots \\
a_{1 N} b_{1}+a_{2 N} b_{2}+\cdots+\left(a_{N N}-\rho\right) b_{N}=0
\end{array} .\right.
$$

Ulteriorly, Eq. 26 can be rewritten as follows

$$
\boldsymbol{A}_{\boldsymbol{T}} \cdot(\boldsymbol{B}-\rho \boldsymbol{I})=\mathbf{0},
$$

by assuming

$$
\boldsymbol{A}_{\boldsymbol{T}}=\left[\begin{array}{c}
a_{11}, a_{21}, \cdots, a_{N 1} \\
a_{12}, a_{22}, \cdots, a_{N 2} \\
\vdots \\
a_{1 N}, a_{2 N}, \cdots, a_{N N}
\end{array}\right]=\left[\begin{array}{c}
p_{11}(T), p_{21}(T), \cdots, p_{N 1}(T) \\
p_{12}(T), p_{22}(T), \cdots, p_{N 2}(T) \\
\vdots \\
p_{1 N}(T), p_{2 N}(T), \cdots, p_{N N}(T)
\end{array}\right]
$$

and

$$
\boldsymbol{B}=\left(b_{1}, b_{2}, \cdots, b_{N}\right)^{\mathrm{T}},
$$

where $\rho$ is the eigenvalue of the matrix $\boldsymbol{A}_{\boldsymbol{T}}$, which means that if and only if all

$|\rho|<1$ the zero solution is asymptotically stable, otherwise the zero solution is unstable.

\section{Dynamical Analysis}

\subsection{General Method for Stability Analysis of System}

The motion equation (1) is rewritten into a first-order form, in order to analyze the periodic stability of formation system

$$
\dot{\boldsymbol{x}}=\boldsymbol{F},
$$


where

$$
\begin{gathered}
\boldsymbol{x}=\left(u_{1}, v_{1}, w_{1}, \cdots, u_{n+m}, v_{n+m}, w_{n+m}, x_{1}, y_{1}, z_{1}, \cdots, x_{n+m}, y_{n+m}, z_{n+m}\right)^{\mathrm{T}}, \\
\boldsymbol{F}=\left(f_{1}^{x}, f_{1}^{y}, f_{1}^{z}, \cdots, f_{n+m}^{x}, f_{n+m}^{y}, f_{n+m}^{z}, u_{1}, v_{1}, w_{1}, \cdots, u_{n+m}, v_{n+m}, w_{n+m}\right)^{\mathrm{T}}, \\
\left(u_{i}, v_{i}, w_{i}\right)^{\mathrm{T}}=\dot{\boldsymbol{r}}_{\boldsymbol{i}},
\end{gathered}
$$

and

$$
\left(f_{i}^{x}, f_{i}^{y}, f_{i}^{z}\right)^{\mathrm{T}}=\boldsymbol{G}_{\boldsymbol{i}}+\boldsymbol{T}_{\boldsymbol{i}}+\boldsymbol{D}_{\boldsymbol{i}}+\boldsymbol{F}_{\boldsymbol{i}}^{e}+\boldsymbol{F}_{\boldsymbol{i}}^{\boldsymbol{k}},
$$

Assume $P_{0}:(\overline{\boldsymbol{x}})$ is a fixed point on the Poincare section, where

$$
\overline{\boldsymbol{x}}=\left(\bar{u}_{1}, \bar{v}_{1}, \bar{w}_{1}, \cdots, \bar{u}_{n+m}, \bar{v}_{n+m}, \bar{w}_{n+m}, \bar{x}_{1}, \bar{y}_{1}, \bar{z}_{1}, \cdots, \bar{x}_{n+m}, \bar{y}_{n+m}, \bar{z}_{n+m}\right)^{\mathrm{T}},
$$

$\boldsymbol{p}=\left(p_{1}, \cdots, p_{6(n+m)}\right)^{\mathrm{T}}$ is $2(n+m)$ small amounts, $P_{1}:(\overline{\boldsymbol{x}}+\boldsymbol{p})$ is a point in the small neighborhood of $P_{0}:(\overline{\boldsymbol{x}})$, so $P_{1}$ can be regarded as a perturbation of $P_{0}$. Therefore, $P_{1}$ can be expanded into Taylor series form by retaining the linear terms based upon $P_{0}$, which can be obtained as follows

$$
\dot{p}=J_{0} \cdot p
$$

where

$$
\boldsymbol{J}_{\mathbf{0}}=\left.\frac{\partial \boldsymbol{F}}{\partial \boldsymbol{x}^{\mathrm{T}}}\right|_{\boldsymbol{x}=\overline{\boldsymbol{x}}} .
$$

The system (38) with the initial condition

$$
\left\{\begin{array}{l}
p_{1}\left(t_{0}\right)=1, p_{2}\left(t_{0}\right)=0, \cdots, p_{6(n+m)}\left(t_{0}\right)=0 \\
p_{1}\left(t_{0}\right)=0, p_{2}\left(t_{0}\right)=1, \cdots, p_{6(n+m)}\left(t_{0}\right)=0 \\
\vdots \\
p_{1}\left(t_{0}\right)=0, p_{2}\left(t_{0}\right)=0, \cdots, p_{6(n+m)}\left(t_{0}\right)=1
\end{array},\right.
$$

can be solved by dividing into the following two cases, based on the ordinary 90 differential equation theory. 
1. When the matrix $\boldsymbol{J}$ has $N=6(n+m)$ linearly independent eigenvectors $\boldsymbol{v}_{\mathbf{1}}, \boldsymbol{v}_{\mathbf{2}}, \cdots, \boldsymbol{v}_{\boldsymbol{N}}$, and their corresponding eigenvalues are $\boldsymbol{\lambda}_{\mathbf{1}}, \boldsymbol{\lambda}_{\mathbf{2}}, \cdots, \boldsymbol{\lambda}_{\boldsymbol{N}}$, then a basis solution matrix of homogeneous linear differential equations with constant coefficients Eq. (30) can always be expressed as

$$
\boldsymbol{P}(t)=\left(\boldsymbol{p}_{\mathbf{1}}(t), \boldsymbol{p}_{\mathbf{2}}(t), \cdots, \boldsymbol{p}_{\boldsymbol{N}}(t)\right)=\left(e^{\lambda_{1} t} \boldsymbol{v}_{\mathbf{1}}, e^{\lambda_{2} t} \boldsymbol{v}_{\mathbf{2}}, \cdots, e^{\lambda_{N} t} \boldsymbol{v}_{\boldsymbol{N}}\right)
$$

the general solution can be expressed as

$$
\boldsymbol{p}(t)=\boldsymbol{P}(t) \cdot \boldsymbol{C}
$$

where $\boldsymbol{C}=\left(C_{1}, C_{2}, \cdots, C_{N}\right)^{\mathrm{T}}$ is undetermined constants matrix. Substituting the initial condition 38 into Eq. (40), the equation can be obtained as follows

$$
I=V \cdot C
$$

where

$$
\boldsymbol{V}=\left(\boldsymbol{v}_{\mathbf{1}}, \boldsymbol{v}_{\mathbf{2}}, \cdots, \boldsymbol{v}_{N}\right),
$$

is eigen-matrix of $\left.J\right|_{\boldsymbol{x}=\overline{\boldsymbol{x}}}$, and the constant matrix satisfies the relation as follows

$$
\boldsymbol{C}=\boldsymbol{V}^{-1}
$$

Thus, the general solution can be rewritten as

$$
\boldsymbol{p}(t)=\boldsymbol{P}(t) \cdot \boldsymbol{V}^{-1} .
$$

2. When the matrix $\boldsymbol{J}$ has less than $N$ linearly independent eigenvectors, it is easy to prove the following conclusion. If $\lambda_{i}$ is the $N_{i}$-fold eigenvalue of $\boldsymbol{J}$, then there must be $N_{i}$ linearly independent solutions in the form of

$$
\boldsymbol{p}(t)=e^{\lambda_{i} t} \sum_{j=0}^{N_{i}-1} \frac{t^{j}}{j !} \boldsymbol{v}_{\boldsymbol{i}, \boldsymbol{j}},
$$

in which $\boldsymbol{v}_{i, 0}$ is a non-zero solution of

$$
\left(\boldsymbol{J}_{\mathbf{0}}-\lambda_{i} \boldsymbol{I}\right)^{N_{i}} \boldsymbol{v}=\mathbf{0},
$$


and a recurrence relation can be obtained as follows

$$
\boldsymbol{v}_{i, j}=\left(\boldsymbol{J}_{\mathbf{0}}-\lambda_{i} \boldsymbol{I}\right) \boldsymbol{v}_{i, j-1},
$$

where $1 \leq j \leq N_{i}-1$. Define the matrix $\boldsymbol{P}$ and $\boldsymbol{V}$ is consisted of $N$ linearly independent vectors $\boldsymbol{p}_{\boldsymbol{i}}$ and $\boldsymbol{v}_{\boldsymbol{i}}$, then the general solution of the system in this situation can be solved by substituting $\boldsymbol{P}$ and $\boldsymbol{V}$ into (44).

The eigenvalues of the matrix $\boldsymbol{p}(t)$ at the periodic sampling time $\rho_{1}, \rho_{2}, \cdots, \rho_{N}$ are the Floquet multiples, according to the Floquet Theory in subsection 2.2 It is easy to prove that the Floquet multiplier of the system is the eigenvalue of the matrix

$$
\boldsymbol{A}_{\boldsymbol{T}}=\boldsymbol{p}(T)
$$

by assuming $t_{0}=0$, and the periodic solution must be asymptotically stable if and only if the condition is satisfied

$$
\left|\rho_{i}\right|_{\max }<1, \quad 1 \leq i \leq N
$$

\subsection{Discussions of Numerical Results}

Taking a 3-star system connected by massless tethers as an example, and the stability analysis is carried out based on the above general model and analysis method, by letting

$$
\left\{\begin{array}{l}
n=3 \\
m=0
\end{array} .\right.
$$

The motion equation of the system can be obtained, by assuming the initial position and velocity is in the xoy plane, and the details can be seen in Appendix. Considering that the system has an initial steady-state configuration of regular $N$-polygon, which may be assumed that the 1st satellite is located on the $y$ axis at the initial moment, and the distance from the origin of the $o-x y z$ is $R_{0}$. 
Then the initial position of any satellite can be obtained as follows

$$
\left\{\begin{array}{l}
\bar{x}_{i}=R_{0} \cos \left((i-1) \delta \alpha+\frac{\pi}{2}\right) \\
\bar{y}_{i}=R_{0} \sin \left((i-1) \delta \alpha+\frac{\pi}{2}\right) \\
\bar{z}_{i}=0 \\
\bar{u}_{i}=-\omega R_{0} \sin \left((i-1) \delta \alpha+\frac{\pi}{2}\right) \\
\bar{v}_{i}=\omega R_{0} \cos \left((i-1) \delta \alpha+\frac{\pi}{2}\right) \\
\bar{w}_{i}=0
\end{array}\right.
$$

in which $\delta \alpha=\frac{2 \pi}{N}$ expresses the angle between two adjacent satellites. Since the size of the tethered satellite system is much smaller than its orbital radius, the gravity term can be approximated as follows

$$
-\frac{\mu_{E} L_{0}}{2\left(\frac{L_{0}^{2}}{4}+R^{2}\right)^{\frac{3}{2}}} \approx-\frac{\mu_{E} L_{0}}{2 R^{3}} .
$$

It is easy to prove that $R_{0}$ satisfies the following relation

$$
-\omega^{2} R_{0}=\left.\dot{u}_{1}\right|_{t=0}
$$

In order to verify the correctness of the initial steady-state configuration of tethered satellite system, a simulation model was established. In the Fig. 3. and Fig. 4 trajectories of the 1st, 2nd, and 3rd satellites are represented by red, green, and blue dotted curves, respectively, the black sphere represents each satellite, and the solid black lines represent the tethers that connects the two adjacent satellites, under the spin angular velocity condition $|\omega|=2.0 \Omega$ and $|\omega|=2.5 \Omega$, separately. Based on the motion equation (56) and the initial conditions (51), the tether system can maintain the initial configuration depend on its spin under both conditions, which proves the correctness of the steadystate configuration and the solution of the initial conditions.

Substituting Eq. $\sqrt{52}$ and the initial conditions into the motion equation 


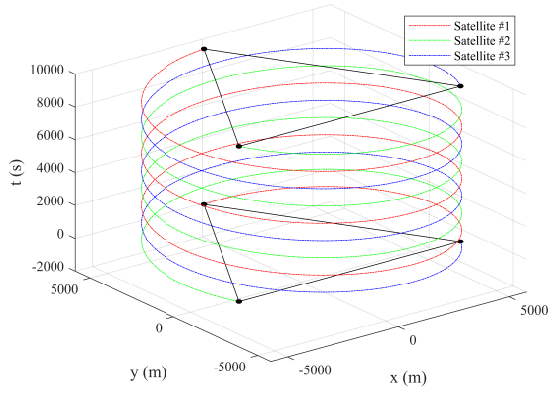

(b)

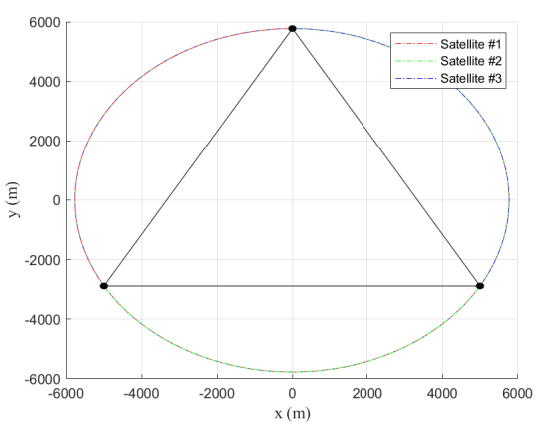

Figure 3: Clockwise spin angular velocity $|\omega|=2.0 \Omega$ of tether formation system, the numerical simulation results of the trajectories of each satellite.
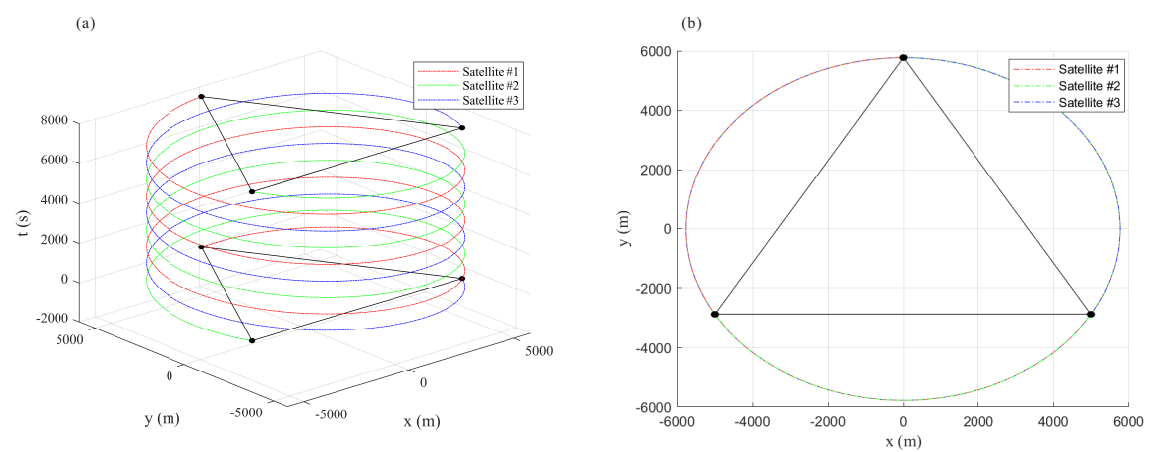

Figure 4: Clockwise spin angular velocity $|\omega|=2.5 \Omega$ of tether formation system, the numerical simulation results of the trajectories of each satellite.

(56), the Jacobian matrix can be solved as follows

$$
\boldsymbol{J}_{\mathbf{0}}=\left.\left[\begin{array}{llll}
\frac{\partial \dot{u}_{1}}{\partial u_{1}}, & \frac{\partial \dot{u}_{1}}{\partial v_{1}}, & \cdots, & \frac{\partial \dot{u}_{1}}{\partial z_{3}} \\
\frac{\partial \dot{v}_{1}}{\partial u_{1}}, & \frac{\partial \dot{v}_{1}}{\partial v_{1}}, & \cdots, & \frac{\partial \dot{v}_{1}}{\partial z_{3}} \\
\vdots & \vdots & \ddots, & \vdots \\
\frac{\partial \dot{z}_{3}}{\partial u_{1}}, & \frac{\partial \dot{z}_{3}}{\partial v_{1}}, & \cdots, & \frac{\partial \dot{z}_{3}}{\partial z_{3}}
\end{array}\right]\right|_{t=0}=\left[\begin{array}{ll}
\boldsymbol{A}, & \boldsymbol{B} \\
\boldsymbol{I}, & \mathbf{0}
\end{array}\right] .
$$

The curve of Floquet multiplier depend on clockwise spin angular velocity of the tethered satellite system can be obtained based upon the method mentioned in subsection 3.1 with the parameters shown in Table 1 It can be found from FIG. 5 that the configuration is stable when the spin angular velocity is higher 
Table 1: Parameters of the tethered satellite system

\begin{tabular}{ccc}
\hline \hline Physical Significance & Symbol & Value \\
\hline Radius of the earth & $R_{E}$ & $6378 \mathrm{~km}$ \\
Mass of the Satellite & $M$ & $100 \mathrm{~kg}$ \\
Equilibrium length of tether & $L$ & $10 \mathrm{~km}$ \\
Young's modulus of tether & $E$ & $5 \times 10^{10} \mathrm{Nm}^{-2}$ \\
Cross sectional area of tether & $A$ & $1 \times 10^{-6} \mathrm{~m}^{2}$ \\
Orbit radius of revolution & $H$ & $1000 \mathrm{~km}$ \\
\hline \hline
\end{tabular}

than the critical angular velocity (about 2.1 times of the revolution angular velocity). The correctness of the critical angular velocity solved by the Floquet
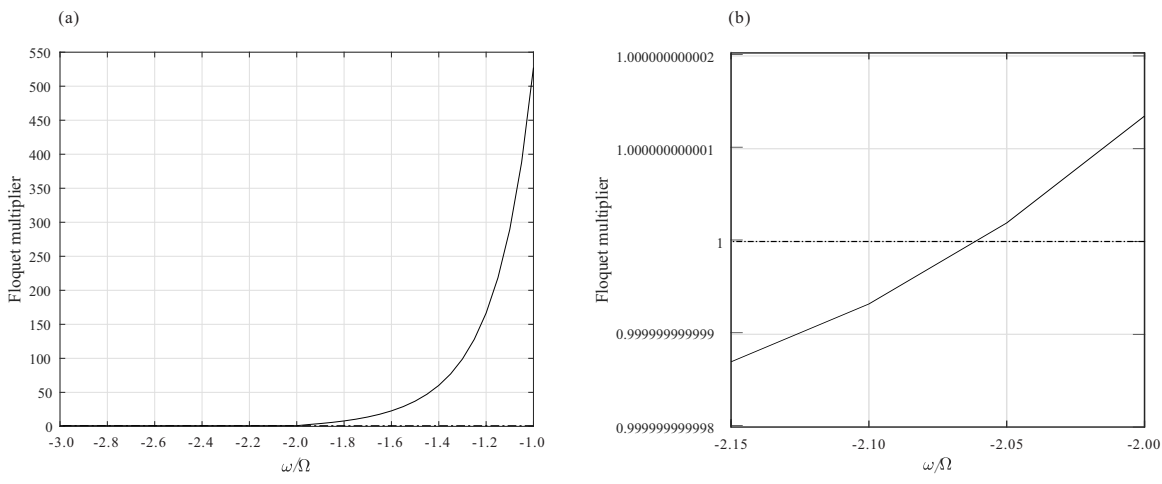

Figure 5: The Floquet Multiplier of the system in clockwise spin, (a) for the curve of Floquet multiplier and spin angular velocity, (b) for the local enlargement of the curve.

110

theory can be proved by introducing disturbances into the systems with different spin angular velocity as shown in FIG. 3 and FIG. 4 respectively. The operation situation of the disturbed system with the spin angular velocity of $|\omega|=2.0 \Omega$ and $|\omega|=2.5 \Omega$ is shown in FIG. 6 and FIG. 7, respectively. Obviously, the configuration of the disturbed system can not be held when the spin angular velocity is lower than the critical angular velocity $\left(\left|\frac{\omega}{\Omega}\right|=2.0<2.1\right)$, while the disturbed system will converge to the original configuration in about 2 periods, when the spin angular velocity satisfies $\left|\frac{\omega}{\Omega}\right|=2.5>2.1$. 


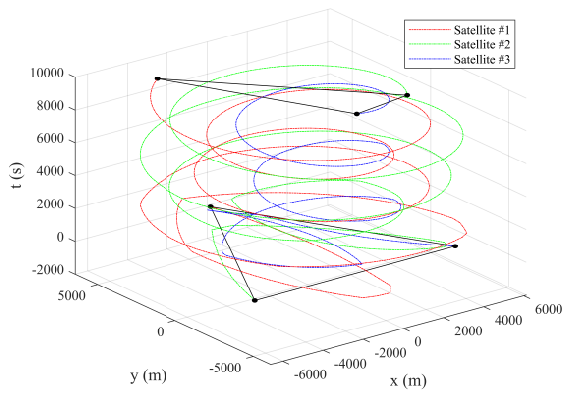

(b)

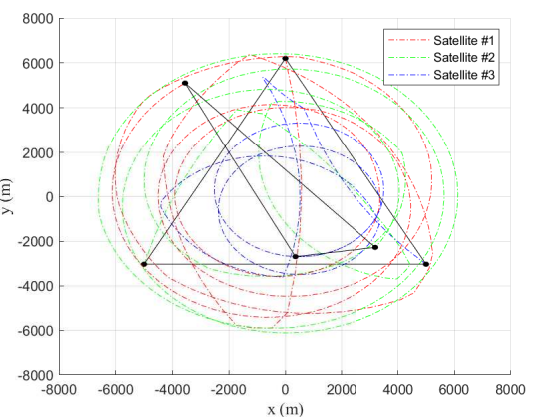

Figure 6: The orbit of each satellite, when the clockwise spin angular velocity of the system satisfies $|\omega|=2.0 \Omega$.

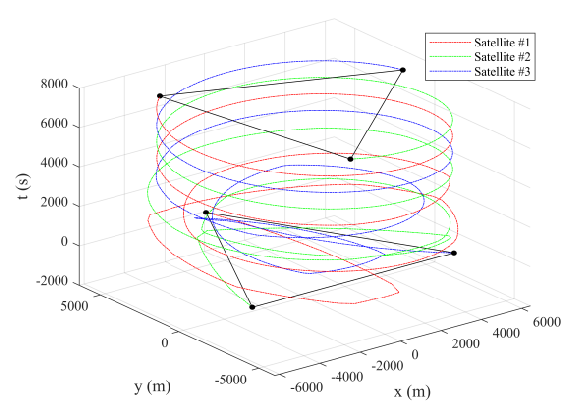

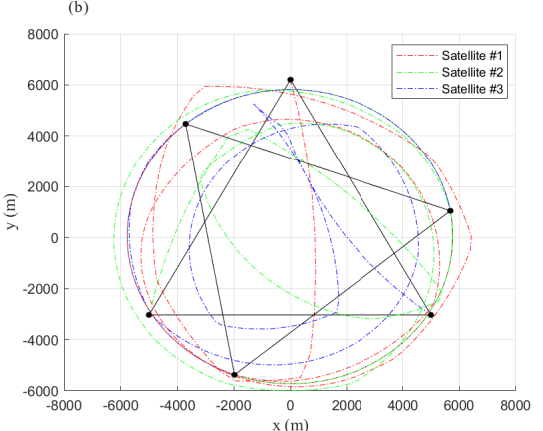

Figure 7: The orbit of each satellite, when the clockwise spin angular velocity of the system satisfies $|\omega|=2.5 \Omega$.

The relative deviation from the initial steady-state configuration is defined as follows

$$
d_{i}=\frac{\left|\boldsymbol{r}_{\boldsymbol{i}}\right|}{R_{0}}-1
$$

to analyse the convergence of each satellite to the initial configuration depends on time. The curves of two conditions with the spin angular velocity of $|\omega|=2.0 \Omega$ and $|\omega|=2.5 \Omega$ are shown in FIG. 8. It is shows that the relative deviation of each satellite in the disturbed system cannot be attenuated to 0 when the spin angular velocity is less than critical angular velocity, shown in FIG. 8 (a); but the relative deviation of each satellite will gradually decay to 0 to restore the initial steady state configuration in a limited time, when the 

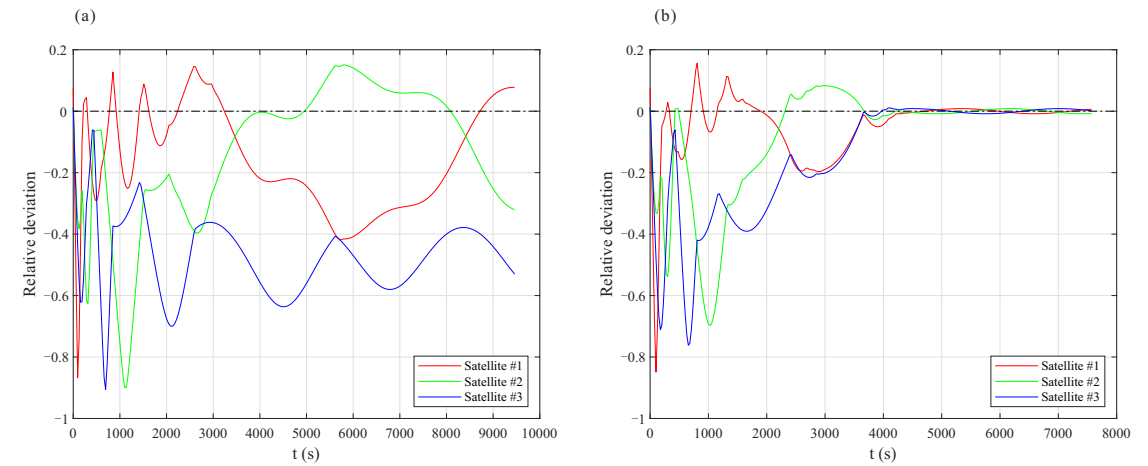

Figure 8: Relative deviation of the system, (a) for the situation of $|\omega|=2.0 \Omega$, and (b) for the situation of $|\omega|=2.5 \Omega$.

spin angular velocity satisfies $\left|\frac{\omega}{\Omega}\right|=2.5>2.1$. Therefore, the solution of periodical stability obtained by the general method based upon the Floquet theory mentioned in subsection 3.1, which the equilateral triangle configuration is a spin-stable configuration when the clockwise spin angular velocity greater than about 2.1 times the revolution angular velocity, has been proved.

\section{Conclusion and Outlooks}

Firstly, in this paper, a general dynamic model of $n$-star tethered satellite system with closed-loop configuration has been proposed. An analytical method of periodic solution stability of the general dynamic model is provided based on Floquet theory, which proved that the periodic solution stability of the system depends on the maximum modulus of the eigenvalue for a matrix related to the Jacobian matrix of the system. On the basis of the general periodic solution stability analysis method, taking a 3-star system with the initial configuration of equilateral triangle, the critical stable clockwise spin angular velocity of the system is analyzed, and the numerical simulation is carried out to verify the results. The results show that the analytical method of periodic solution stability can solve the critical stable spin angular velocity of the tethered satellite system accurately, and the 3-star system can guarantee stable spin when the value of 
its clockwise spin angular velocity is higher than 2.1 times of revolution angular velocity, otherwise the disturbed system will not be able to re-converge to the initial configuration in finite time.

\section{Acknowledgements}

The first and the third author acknowledges the financial support from Natural Science Foundation of China (Key Project Grant Nos. 11732006), the fourth author acknowledges the financial support from Natural Science Foundation of China (Key Project Grant Nos. 91848205), and the other authors acknowledges the financial support from Natural Science Foundation of China (Grant Nos. 12072263, 11802235), Natural Science Foundation of Shaanxi Province (Grant No. 2020JQ-129) and State Key Laboratory of Mechanical Behavior and System Safety of Traffic Engineering Structures (Grant No. KF2020-26).

\section{Data Availability}

Data sharing not applicable to this article as no datasets were generated or analysed during the current study.

\section{Competing financial interests}

The authors declare no competing financial interests. 


\section{Appendix}

The motion equation of the 3-star system can be obtained as follows

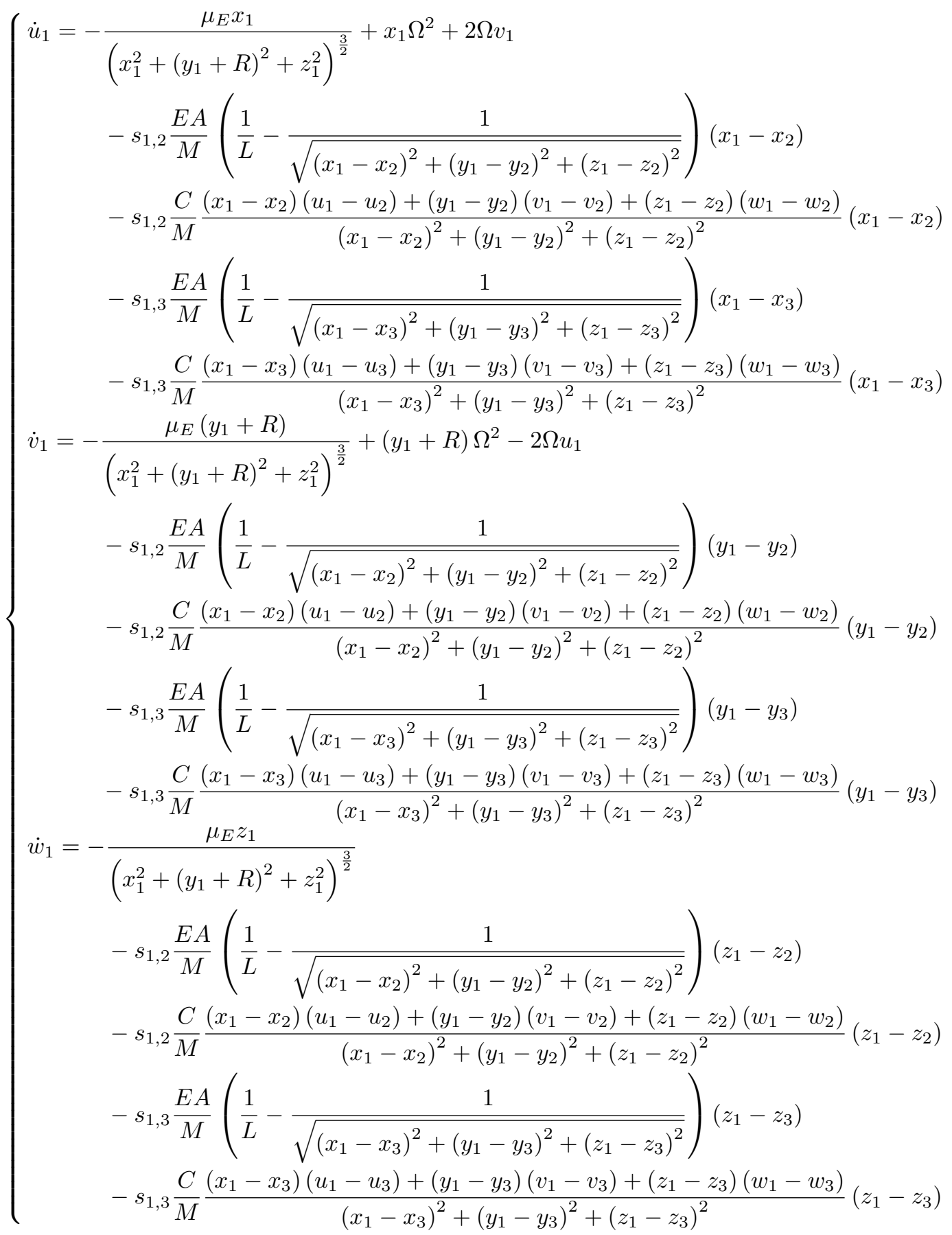




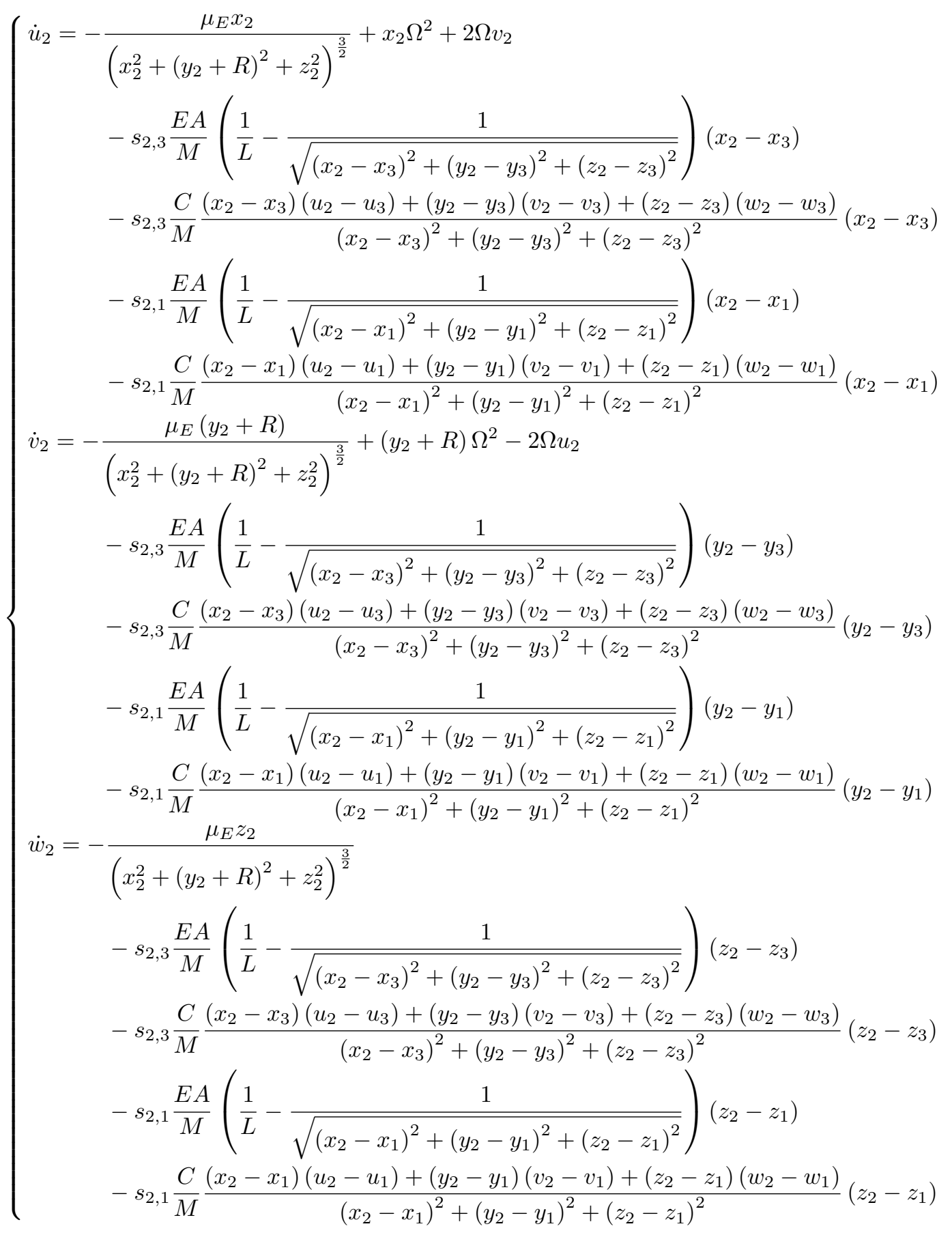




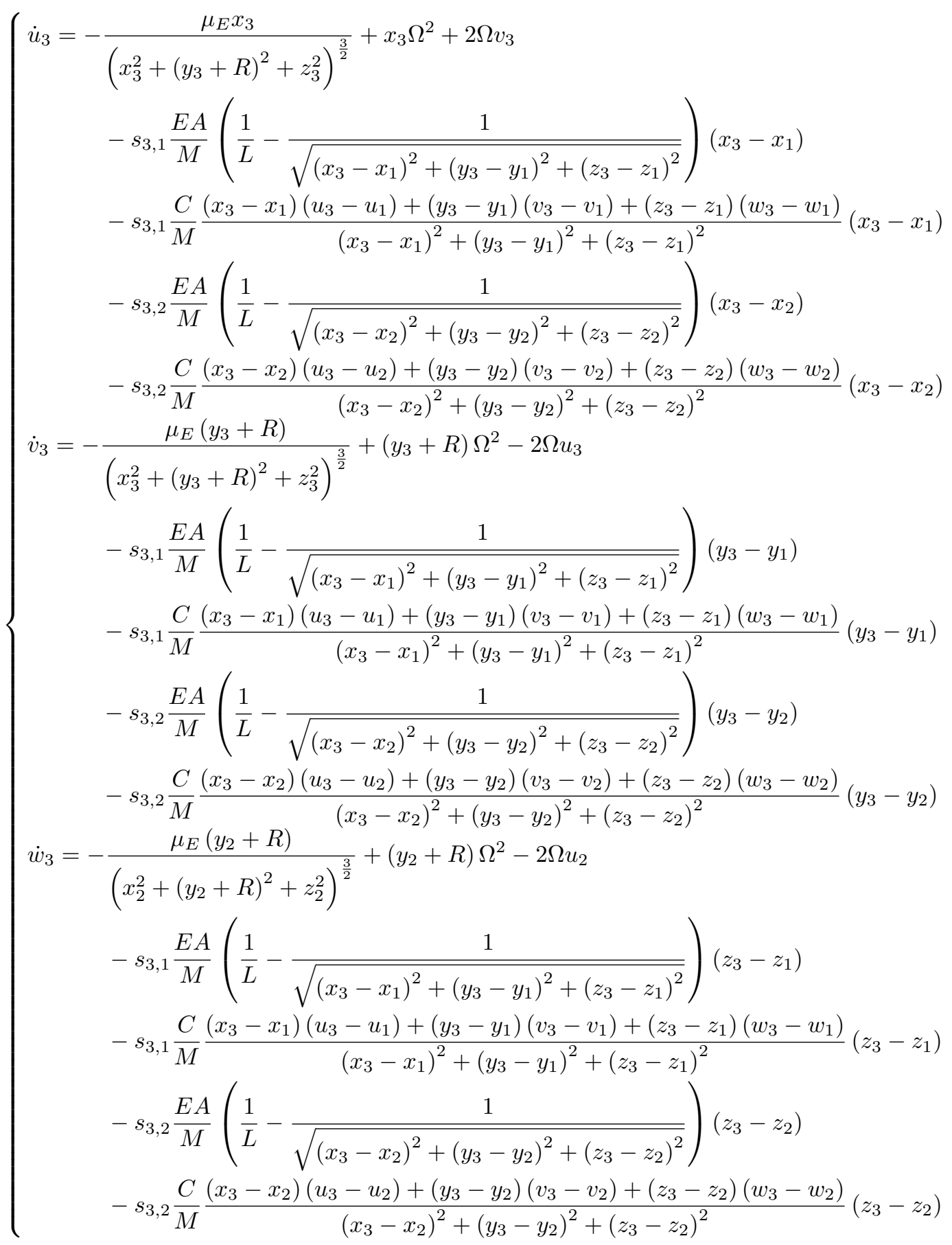




$$
\left\{\begin{array}{l}
\dot{x}_{1}=u_{1} \\
\dot{y}_{1}=v_{1} \\
\dot{z}_{1}=w_{1} \\
\dot{x}_{2}=u_{2} \\
\dot{y}_{2}=v_{2} \\
\dot{z}_{2}=w_{2} \\
\dot{x}_{3}=u_{3} \\
\dot{y}_{3}=v_{3} \\
\dot{z}_{3}=w_{3}
\end{array}\right.
$$

where $m_{i}=M, E_{i, j}=E, A_{i, j}=A, C_{i, j}=C, L_{i, j}=L$, and $i=1,2,3$.

\section{References}

[1] Wen, H., Jin, D. \& Hu, H. Advances in dynamics and control of tethered satellite systems. Acta Mech Sin 24, 229 (2008)

[2] Kumar, K. Review of dynamics and control of nonelectrodynamic tethered satellite systems. J. Spacecr. Rockets 38 (12), 705 - 720 (2006)

[3] Huang, P., Zhang, F., Cai, J., et al. Dexterous tethered space robot: design, measurement, control and experiment. IEEE Trans. Aerosp. Electron. Syst. $53(3), 1452-1468$ (2017)

[4] Shan, M., Guo, J., Gill, E. Review and comparison of active space debris capturing and removal methods. Prog. Aero. Sci. 80, 18 - 32 (2016)

[5] Zhang, F., Huang, P. Releasing dynamics and stability control of maneuverable tethered space net. IEEE ASME Trans. Mechatron. 22 (2), 983 993(2017) 
[6] Dobrowolny, M., N. H. Stone. A technical overview of TSS-1: The firest Tethered-Satellite system mission. Il Nuovo Cimento C, 1994. 17(1): p. $1-12$.

[7] Chen H., Wen H., Jin D., et al. Experimental studies on tethered satellite systems [J]. Advances in Mechanics, 2013, 43(1): 174-184.

[8] Carroll J. A., Oldson J. C. Tethers for small satellite applications. In: AIAA/USU Small Satellite Conference, Logan, Utah, 1995.

[9] Mccoy J. E., C. ONeill J. S., Settecerri T., et al. Plasma motor-generator (PMG) flight experiment results. In: Proceedings of the 4th International conference on Tethers in Space, Washington DC, 1995.

[10] Laframboise J. G., Wallis D. D., James H. G. Effects of large-amplitude RF emissions on OEDIPUS-C floating voltages. In: 8th Spacecraft Charging Technology Conference, English, 2004.

[11] James P. Hypervelocity impact studies on space tethers. In: 54th International Astronautical Congress of the International Astronautical Federation (IAF). Bremen, Germany,2003.

[12] Cosmo M. L., Lorenzini E. C. Tether in space handbook. Huntsville, AL: Smithsonian Astrophysical Observatory, NASA Marshall Space Flight Center, 1997.

[13] Koss S. Tether Deployment mechanism for the advanced tether experiment (ATEx). In: Proceeding 7th European Space Mechanisms \& Tribology Symposium. ESTEC, No-ordwijk, The Netherlands, 1997. 175-182.

[14] Fan, G., Zhang, Y., Huang, P., et al. State estimation of double-pyramid tethered satellite formations using only two GPS sensors. Acta Astronaut. $180,507-515(2021)$

[15] Yu, B., Jin, D., Wen, H. Analytical deployment control law for a flexible tethered satellite system. Aero. Sci. Technol. 66, 294 - 303 (2017) 
[16] Ma, Z., Sun, G., Li, Z. Dynamic adaptive saturated sliding mode control for deployment of tethered satellite system. Aero. Sci. Technol. 66, 355 $365(2017)$

[17] Liu, H., He, Y., Yan, H., et al. Tether tension control law design during orbital transfer via small-gain theorem. Aero. Sci. Technol. 63, 191 202(2017)

[18] Wen, H., Zhu, Z., Jin, D., et al. Space tether deployment control with explicit tension constraint and saturation function. J. Guid. Control Dyn. 39 (4), 915 C 920

[19] Sakawa, Y., Shindo, Y. Optimal control of container cranes. Automatica18 (3), $257-266(1982)$

[20] Singhose, W., Kim, D., Kenison, M. Input shaping control of doublependulum bridge crane oscillations. J. Dyn. Syst. Trans. ASME 130 (3),1 $-7(2008)$

[21] Williams, P. Optimal control of a spinning double-pyramid Earth-pointing tethered formation. Acta Astronaut. 64, 1191 - 1223 (2009)

[22] Wang, C., Zhang, F. Finite-time stability of an underactuated tethered satellite system. Acta Astronaut. 159, 1191 - 1223 (2009)

[23] Nayfeh A. H., Mook D. T. Nonlinear Oscillations [M]. New York: WileyInterscience, 1995, 26-30, 273-302, 365-366.

[24] Teschl G. Ordinary Differential Equations and Dynamical Systems [M]. Lecture Notes, University of Vienna, 2000, 65-76.

225 [25] Moser J. Convergent Series Expansions for Quasi-Periodic Motions [J]. Mathematische Annalen, 1967, 167(1): 136-176.

[26] Guido G, Daniel A C, Joao C A. Stability for Quasi-Periodically Perturbed Hills Equations [J]. Communications in Mathematical Physics, 2005, 260: 403-443. 
[27] Zhang Z. Birurcations and hysteresis of varying compliance vibrations of a ball bearing-rotor system [D]. Harbin Institute of Technology, 2015.

[28] Yang R. Research on dynamic characteristics and its application of a bal1 bearing-rigid rotor system with a local defect [D]. Harbin Institute of Technology, 2018. 